\title{
APLICACIÓN DE LA METÁFORA BIOLÓGICA PARA EL DESARROLLO DE FORMAS ORGANIZATIVAS EN LA INTEGRACIÓN EMPRESARIAL*
}

\author{
LUZ ALEXANDRA MONTOYA RESTREPO** \& IVÁN ALONSO MONTOYA RESTREPO **** \\ UNIVERSIDAD NACIONAL DE COLOMBIA
}

Recibido/ Received/ Recebido: 07/04/2011 - Aceptado/ Accepted / Aprovado: 01/07/2012

\begin{abstract}
Resumen
La capacidad de las empresas de adaptarse en el mercado no depende solamente de la competencia, puede alcanzarse a partir de mecanismos de cooperación interempresarial, permitiéndole ventajas importantes a las pequeñas organizaciones nacientes. Un ejemplo muy didáctico de este proceso lo ofrece la naturaleza. El presente documento toma como metodología principal el desarrollo de la metáfora biológica para, demostrar cómo mediante la cooperación y la co-evolución inter-empresariales posible que empresas pequeñas puedan alcanzar ventajas competitivas. Se señala la trascendencia de que los nuevos empresarios entiendan formas organizacionales novedosas de cooperación y co-evolución derivadas de modelos biológicos exitosos y de la importancia de su adaptación a ellas de tal forma que surjan nuevas formas empresariales.

Palabras clave: Ecosistemas organizacionales, Cooperación, Co-evolución, Integración empresarial.

\section{APPLICATION OF BIOLOGICAL METAPHOR FOR THE DEVELOPMENT OF ORGANIZATIONAL FORMS IN ENTREPRENEURIAL INTEGRATION}

\begin{abstract}
Enterprise capacity to adapt to the market does not depend only on competence; it can be achieved using inter-entrepreneurial cooperation mechanisms, allowing important advantages to small new organizations. An example of this process is offered by nature. This study uses as main methodology the development of biological metaphor to demonstrate how through cooperation and interentrepreneurial co-evolution is possible that small companies achieve competitive advantages. The transcendence that new entrepreneurs understand new organizational forms of cooperation and co-evolution from successful biological models and the importance of their adaptation so new entrepreneurial forms can be arise.
\end{abstract}

Keywords: Organizational ecosystems, Cooperation, Co-evolution, Entrepreneurial integration.

Artículo de reflexión derivado del proyecto de investigación titulado "Metáforas biológicas y organización", financiado por la Universidad Nacional de Colombia, código Hermes № 11442 (Sistema de información de Investigación, Vicerrectoría de Investigación).

*** Coordinadora Nacional del Concurso Docente, Universidad Nacional de Colombia, Profesora Asociada, Facultad de Ciencias Económicas, Doctora en ciencias Económicas, Magister en Administración, Administradora de empresas. Universidad Nacional de Colombia Edificio Uriel Gutiérrez. Correo electrónico: lamontoyar@unal.edu.co

**** Profesor Asociado, Facultad de Minas, Doctor en Ciencias Económicas, Magister en Administración, Administrador de empresas. Universidad Nacional de Colombia (Medellín). Correo electrónico: iamontoyar@unal.edu.co 


\title{
APLICAÇÃO DA METÁFORA BIOLÓGICA PARA O DESENVOLVIMENTO DE FORMAS ORGANIZATIVAS NA INTEGRAÇÃO EMPRESARIAL
}

\author{
Resumo
}

\begin{abstract}
A capacidade das empresas de adaptar-se ao mercado não depende somente da concorrência. Isto pode ser conseguido também a partir de mecanismos de cooperação interempresarial, permitindo vantagens importantes às pequenas organizações nascentes. Um exemplo muito didático deste processo oferece a natureza. O presente documento toma como metodologia principal o desenvolvimento da metáfora biológica para demonstrar como mediante a cooperação e a coevolução interempresariais é possível que pequenas empresas possam atingir vantagens competitivas. Assinala-se a importância de que os novos empresários compreendam formas organizacionais inovadoras de cooperação e de coevolução derivadas de modelos biológicos exitosos e da importância de sua adaptação a elas de tal forma que surjam novas formas empresariais.
\end{abstract}

Palavras chave: Ecossistemas organizacionais, Cooperação, Coevolução, integração empresarial.

Montoya, L. \& Montoya, I. (2012) Aplicación de la metáfora biológica para el desarrollo de formas organizativas en la integración empresarial. En: Revista de la Facultad de Ciencias Económicas de la Universidad Militar Nueva Granada. rev.fac.cienc.econ, XX (2).

JEL: M11, M29.

\section{Introducción}

Puede indicarse que se ha alcanzado un consenso asistido por la Conferencia de las Naciones Unidas sobre Comercio y Desarrollo (2010) y por los diferentes instrumentos de medición de la competitividad comúnmente aceptados por los países (Foro Económico Mundial, 2010; International Institute for Management Development, 2010; Sistema Nacional De Competitividad, 2008) sobre los beneficios que aporta la integración en formas empresariales a los problemas de competitividad de las empresas, no sólo de las pymes sino también de las grandes organizaciones, en donde la interacción les permite alcanzar ventajas que de manera aislada no podrían tener (Montoya, 2010; Mincomercio, 2010). Para Porter $(1990 ; 2000)$ la utilización de esquemas productivos y de trabajo integrado en los sectores económicos son las herramientas indispensables para alcanzar ventajas competitivas. En 1995, Bejarano realizo un estudio acerca de la relación entre cadenas productivas y competitividad, señalando cómo las cadenas productivas son la mejor forma de aprehender los elementos del sistema pertinentes para el análisis de la competitividad.
Los beneficios que reporta la integración, entendida esta como la interacción que tienen varias organizaciones para alcanzar un mejor desempeño, dependen entre otros factores de la forma en que se integran, de la capacidad productiva de las empresas y de la competitividad de cada una de ellas de forma que se disminuyan los costos de transacción siendo esto una de las principales motivaciones de la integración (Williamson, 1975).

La intención final de un proceso de integración empresarial (conjunto de relaciones inter-organizacionales) se centra en la promoción de la generación de organizaciones productivas, que adopten esquemas eficientes de relación entre empresas independientes, permitiendo a estas últimas lograr ventajas competitivas que no podrían alcanzar si operaran aisladamente. La respuesta más eficiente para esta problemática se encuentra no solo al interior de las empresas individuales si no en factores externos y colectivos. La colectividad permite a las empresas individuales operar a niveles de eficiencia individuales que de otra forma no podrían alcanzar (Garzón, 2005).

Para alcanzar estos propósitos las organizaciones han desarrollado diferentes mecanismos tales como: 
alianzas financieras, operativas, de mercado, de recursos humanos y administrativas. Este tipo de integraciones han demostrado ser exitosas en contextos internacionales: clúster (Kothandaraman \& Wilson, 2001), distritos industriales (Guerrieri \& Pietrobelli, 2003; Zeriali, 2005), cadenas productivas (Cerdan, 2003), aglomeraciones productivas (Caporali, 2005), mecanismos de desarrollo local (Conzanelli, 2004), entre otros.

Una de las estrategias empresariales más efectivas para operar en el contexto competitivo de negocios es una gestión fuertemente basada en la generación de vínculos de colaboración con otros agentes del entorno cercano. A través de la especialización y colaboración, las empresas pueden acelerar sus procesos de aprendizaje, alcanzar economías de escala y concentrarse en los ámbitos donde poseen mayores ventajas competitivas. En la economía global no son las empresas individuales las que compiten, sino las redes o cadenas de empresas junto a las instituciones con las que interactúan, y que las apoyan en la generación de conocimiento (BID, 2002).

Formas organizacionales alternativas, se hacen necesarias para alcanzar estos objetivos: La capacidad de emprendimiento de los gerentes estará dada por las competencias que estos desarrollen para integrarse y mejorar la competitividad individual y colectiva, en este proceso se hace indispensable la concertación de voluntades, el establecimiento de contratos formales de compromiso, la posibilidad de constituir nuevas formas jurídicas y evitar las prácticas de competencia desleal y oportunismo en el ejercicio de integración (Montoya et al., 2008).

La creación de una red personal se apoya en las relaciones entre empresarios y entre estos y proveedores, inversionistas, amigos, etc., siendo estos contactos quienes pueden ayudarle a tomar decisiones más eficaces, proporcionándole información que reduzca la incertidumbre del negocio. Los empresarios al crear sus propias redes personales, a través de una búsqueda activa de individuos con los mismos intereses, pueden sacarle partido a los nuevos productos o servicios innovadores que otros estén desarrollando. Ya sea a través de las licencias, que proporcionan un acceso limitado a la tecnología, o de las alianzas estratégicas para compartir recursos, un empresario puede tener acceso a expertos de mercadeo y finanzas de las grandes corporaciones $e$ incluso obtener dinero que permita ingresar a nuevos mercados. De esa manera se constituye una red de negocios que es un marco de alianzas creado entre diferentes empresas con el fin de alcanzar objetivos beneficiosos para todos (Velásquez, 2004, 23). De este esquema se encuentra que la integración productiva de las pymes y empresas grandes es posible en la medida en que existan mecanismos de organización de la producción a nivel local y estos se centren en el desarrollo de redes empresariales, las cuales han demostrado ser los motores ideales para impulsar la especialización y el aumento de productividad (CEPAL, 2001; Chavarría, 2002; Farah, 2004).

La cooperación inter-empresarial que logra la eficiencia colectiva se traduce en reducción de costos que eleva la competitividad al igual que eleva las ganancias y los salarios, mejora las condiciones del trabajo y fortalece la flexibilidad y la capacidad de respuesta de la empresa a las condiciones cambiantes de la demanda del mercado (Maya, 2005). La competitividad en el nivel micro está basada en la interacción. Varios autores consideran que para mejorar la capacidad competitiva y lograr una apropiada sinergia es necesaria una base productiva local organizada y estructurada, externalidades derivadas de las economías de aglomeración y el aumento de eficiencia colectiva de una economía de aprendizaje.

\section{Metodología}

La metodología propuesta para el presente análisis se fundamenta en la aplicación de los modelos metafóricos, en este sentido se señalan las dificultades que enfrentan los usuarios de estos mecanismos debido a las aplicaciones simplistas o acomodadas para fortalecer el punto de vista del investigador, pero frente a estas desventajas las posibilidades propuestas las superan en gran magnitud, en especial por su capacidad de entendimiento, por su facilidad de aplicación y en especial por la oportunidad para generar nuevo conocimiento a partir de dichas aplicaciones. La metáfora biológica ha sido ampliamente aceptada (Montoya et al., 2008; Montoya et al., 2009a, 2009b; Montoya et al., 2010a, 2010b, 2010c), en es- 
pecial en el desarrollo de las ciencias económicas, en los modelos evolucionistas y en la teoría de gestión de las organizaciones.

En apoyo a dicha metodología se utilizará en este documento el método de investigación sintético, el cual, como un proceso de razonamiento que tiende a reconstruir un todo a partir de los elementos distinguidos por el análisis, posibilita el realizar una exploración metódica y breve. Este, como un procedimiento mental, que tiene como meta la comprensión total. La síntesis, que significa la reconstrucción y la integración de las partes en un todo, implica una operación analítica (Euler, 2008). Este método permite integrar los elementos en una unidad nueva, en una comprensión total de la esencia, de lo que ya se conoce en todos sus elementos y particularidades (Ortiz \& García, 2003). Se propone entonces la integración de los modelos biológicos con los modelos de integración (formas organizativas), a partir de los ecosistemas organizacionales.

La metáfora, estudiada por Lakoff \& Jhonson (1980; 1999), permite entender cómo, en el proceso de comprensión que ella implica, la experiencia se relaciona con el pensamiento para ser integrada al conocimiento, relación que el lenguaje, a su vez, pone de manifiesto a través de la metáfora. Al utilizar una metáfora son dos o más ideas las que se asocian en la mente del emisor, en virtud de rasgos comunes que, para ese este, quien produce la metáfora, les confieren similitud o parecido, y que le sirven para tender un puente entre dos dominios conceptuales que carecían de conexión (Boquera, 2005).

Morgan (1991), señala diferentes metáforas en el estudio de las organizaciones:

- La organización como máquina, en donde se relaciona con la teoría mecanicista.

- La organización como organismo y como cerebro, que se enfoca en la importancia del procesamiento de la información, el aprendizaje y la inteligencia.

- La organización como cultura, que es vista como la residencia de ideas, valores, normas, rituales y creencias que sostienen la realidad social.
- La metáfora política, que se enfoca en los diferentes conjuntos de intereses, conflictos y juegos potenciales que configuran las actividades organizacionales.

- La organización como una "prisión psíquica", que ve a las personas atrapadas por sus propios pensamientos, ideas y creencias o por preocupaciones originadas en la parte inconsciente de la mente.

- La organización como un instrumento de dominación, que utiliza a sus empleados y los bienes nacionales y mundiales para conseguir sus propios fines.

- La organización vista como un flujo de cambio y transformación.

Una de las principales ventajas de la metáfora biológica es su contribución al entendimiento de las relaciones organización-ambiente. Al enfatizar en las necesidades de supervivencia de la organización mejora su gestión, pues se enfoca en el objetivo principal (misión) y busca el equilibrio de los subsistemas (estratégico, estructural, tecnológico, humano y de dirección) entre sí y con su entorno. Alerta sobre el hecho de que para organizar existe una variedad de opciones y muestra que la organización efectiva depende de las circunstancias del entorno. Con su aporte ecologista refuerza la necesidad de una teoría de las relaciones inter-organizacionales para tratar con entornos complejos, lo cual, a su vez, posibilita la generación de procesos de innovación (Ochoa \& Montoya, 2010).

\section{Formas inter-organizacionales}

Las empresas requieren para su funcionamiento estructuras que les permitan alcanzar sus objetivos, conformando patrones de diseño, las cuales debido a las necesidades se han ido adaptando y evolucionando. Estas estructuras son las formas organizacionales. El desarrollo de la empresa implica actividades estructurales e integradas; es decir, individuos que trabajan juntos o cooperan en relaciones de interdependencia. La noción de interrelación supone un sistema social. Por ello, se puede afirmar que las organizaciones consisten en: i) arreglos orientados a una meta, individuos con un propósito; ii) sistemas psicosociales, individuos que trabajan en grupos; iii) 
sistemas tecnológicos, individuos que utilizan conocimientos y técnicas; y iv) una integración de actividades estructuradas, individuos que trabajan juntos en relaciones estructuradas (Salazar \& Maggiorani, 2005).

Rivas (2002) distingue tres importantes épocas en la evolución de las formas organizacionales, la primera de ellas, la época de la estandarización, que está dominada por la idea de la búsqueda de la especialización, la producción en masa, los controles y el orden para lograr la eficiencia. Este modelo de organización es el inspirado por los autores de la teoría clásica de las organizaciones. A mediados del siglo $\mathrm{XX}$, las empresas automotrices y petroleras experimentaron procesos crecientes de desconcentración y transnacionalización. Esta necesidad de adaptarse a mercados diferenciados obligó a la creación de la segunda época la de la organización divisional, con el propósito de hacer flexible y eficiente la organización. Estas formas se extienden hasta mediados de los años setenta y adoptan formaciones híbridas tales como la organización matricial que permite un enfoque de doble estabilidad: segmentación de mercados emergentes y de clientes.

A partir de los años ochenta, el inicio de la liberalización de los mercados internacionales y la aparición de la globalización, dificulta correlacionar competencias y rutinas organizacionales con oportunidades de mercado, es por ello que aparece la organización en red, favoreciendo la reducción del tamaño y la orientación hacia el negocio central basado en las competencias centrales que demanda el sector industrial. Las redes de firmas proliferan a lo largo de la cadena del valor que de pronto se vuelve virtual en un continuo que rebasa los conceptos tradicionales de espacio y tiempo (Tabla 1).

Rivas (2002) señala como puede apreciarse que este proceso puede verse desde los enfoques organizacional, estructural y estratégico y de acuerdo con ello se ha pasado de las formas intraorganizacionales a las interorganizacionales y de la economía de escala a la de alcance, evolucionando desde la organización jerárquica a la organización de equipos y fortaleciendo las redes estratégicas y las alianzas (Ilustración 1).

Dentro de estas formas que van evolucionando, el surgimiento de nuevas tecnologías de información permite que las formas organizacionales se transformen y se vuelvan más flexibles y que se transformen las estructuras piramidales en otras menos rígidas (Mintzberg, 1995).

\section{Ecosistema organizacional}

El concepto de la "ecología de organización" fue acuñado para confirmar el hecho de que todas las organizaciones son esencialmente sistemas ecológicos complejos caracterizados por la interdependencia social y los cambios de los sistemas físicos. Cuando esta clase de marco de los ecosistemas se aplica a las organizaciones, se puede entender mejor el funcionamiento de organización, incluyendo los aprendizajes informales, examinando todos los componentes y las interdependencias (Becker, 2007). E

Tabla 1. Evolución de los modelos organizacionales ${ }^{1}$

\begin{tabular}{|l|l|l|l|}
\hline \multicolumn{1}{|c|}{ Espacio Histórico } & \multicolumn{1}{|c|}{$\mathbf{1 9 0 0 - 1 9 8 0}$} & \multicolumn{1}{c|}{$\mathbf{1 9 8 1 - 1 9 9 0}$} & \multicolumn{1}{c|}{1991 en adelante } \\
\hline Etapa Histórica & Estandarización & Orientación al cliente & Innovación y desarrollo \\
\hline Estructura organizacional & $\begin{array}{l}\text { Jerarquizada } \\
\text { (Funcional divisional, matricial) }\end{array}$ & Adhocracia, Horizontal en red & Celular, ecológica \\
\hline Activo clave & Bienes de capital & Información & Conocimiento y capacidad de adaptación \\
\hline Gerente clave & Gerente de operaciones & Gerente de Informática & $\begin{array}{l}\text { Gerente de Conocimiento (Chief Knowled- } \\
\text { ge officer CKO) }\end{array}$ \\
\hline Competencias Claves & Especialización y segmentación & $\begin{array}{l}\text { Flexibilidad y capacidad de } \\
\text { respuesta }\end{array}$ & $\begin{array}{l}\text { Diseño de la creatividad y sostenimiento } \\
\text { de relaciones }\end{array}$ \\
\hline
\end{tabular}

3 Fuente: Adaptado de Rivas (2002). 
Ilustración 1. Evolución de las formas organizacionales²

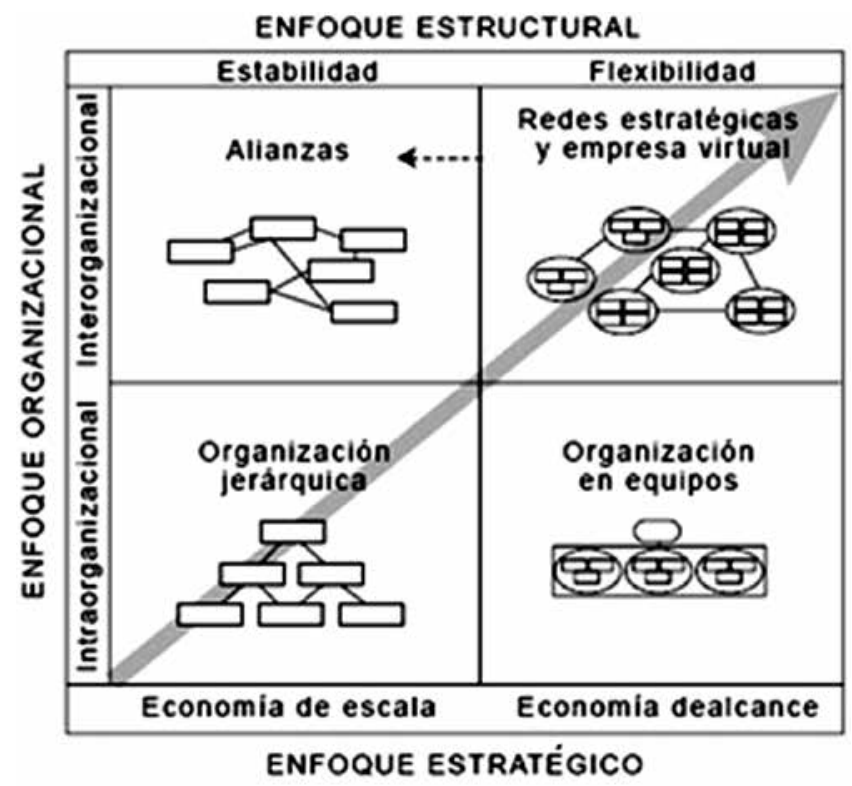

concepto de ecología de organizaciones, fue introducido por Moore, en The Harvard Business Review, al describir una comunidad económica apoyada por la interacción de organizaciones e individuos que pueden ser considerados los organismos del mundo de los negocios. Esta comunidad económica produce bienes y servicios de valor para clientes que son los miembros del ecosistema. Las organizaciones miembros del ecosistema por supuesto incluyen a los proveedores, productores líderes, competidores y otras organizaciones que apoyan. Con el tiempo, ellos codesenvuelven sus capacidades y papeles, y realizan procesos co-evolutivos, y tiende a encuadrarse con las direcciones de las compañías más centrales. Esas compañías que sostienen los papeles de dirección pueden cambiar con el tiempo, pero la función de líder del ecosistema es estimada por la comunidad porque les permite a los miembros, que se acerquen a las visiones compartidas, alinear sus inversiones y encontrar los papeles que se soportan mutuamente (Moore, 1996, 2005). Se señala cómo, para entender el mundo, es útil volver a la biología.
Para los propósitos de esta síntesis sobre el ecosistema organizacional, la biología muestra como los ecosistemas naturales aislados, pueden llegar a ser altamente vulnerables a los desastres ecológicos, y pueden incluso enfrentar las extinciones totales. Es por ello que los de este tipo de ecosistemas (aislados) deben ser protegidos para lograr su sostenibilidad en contraste con los ecosistemas presionados en los que se desarrolla una gran resistencia y flexibilidad, en donde se presenta la co-evolución como la relación benéfica que tienen dos especies para evolucionar. Esta co-evolución puede darse en muchos casos entre miembros de la misma especie, sin embargo, la relación benéfica interespecies, con poblaciones que son totalmente distintas, es la que permite generar mecanismos adaptativos y alcanzar ventajas competitivas que les posibilitan desarrollarse y modificar el medio, evitando la depredación y la competencia (Thompson, 2003). Por su parte Moore (1996; 2005) utiliza la metáfora biológica del ecosistema, al considerarla como una comunidad de organismos que interactúan con otros y el medio ambiente en el cual ellos viven.

2 Fuente: Criado Fernández (2000) citado por Rivas (2002). 
Aplicando los principios de la ecología a los negocios para ayudar a los empresarios y gerentes a entender que la competencia en los negocios ha cambiado en los nuevos tiempos, es importante entender que un ecosistema se convierte en una comunidad de empresas que se relacionan entre sí, más el entorno en el que viven y con el cual también se relacionan (Montoya et al., 2010a). De esta forma se hace la analogía señalando a la sociedad como una comunidad económica está respaldada por una serie de organizaciones e individuos que se relacionan entre sí, los organismos del mundo empresarial, este ecosistema empresarial está formado por clientes, intermediarios del mercado, proveedores y naturalmente cada empresa, las cuales se podrían considerar como las especies primarias del ecosistema. Para triunfar debe desarrollarse una conciencia ecológica, reconociendo que la compañía existe dentro de un ecosistema empresarial y que hay que representar el papel de jardinero, que cuida y moldea el ecosistema mediante su estrategia empresarial (Moore, 1993, 1996), la constitución de un nuevo ecosistema organizacional requiere de la constitución de nuevas formas organizativas que permitan una mejor interacción entre los actores involucrados para alcanzar importantes sinergias, las cuales son requeridas para cooperar y co-evolucionar. Esto será lo que permita que este ecosistema no se convierta en aislado sino en presionado.

El ecosistema de negocios soporta una variedad de industrias y puede ser más rico y más expansivo que las redes de proveedores y empresas extendidas. De esta forma, las compañías que no tienen un ecosistema que permita co-evolucionar alrededor de ideas innovadoras, trabajan cooperativamente para el desarrollo de nuevos productos en busca de satisfacer a los nuevos consumidores y sus necesidades. En este sentido, para que un modelo de ecosistema de negocios sea exitoso es importante que: i) las capacidades fundamentales sean alineadas con los valores de los consumidores; ii) la oferta de productos este basada en las capacidades fundamentales, que pueden generar mayores ventas en volúmenes importantes y economías de escala; iii) experiencia total del consumidor; iv) los beneficios de los productos son reinvertidos en el desarrollo de nuevos productos, el incremento de las capacidades y el soporte de la comunidad y sus alianzas para fortalecer el ecosistema (Ilustración No. 2).

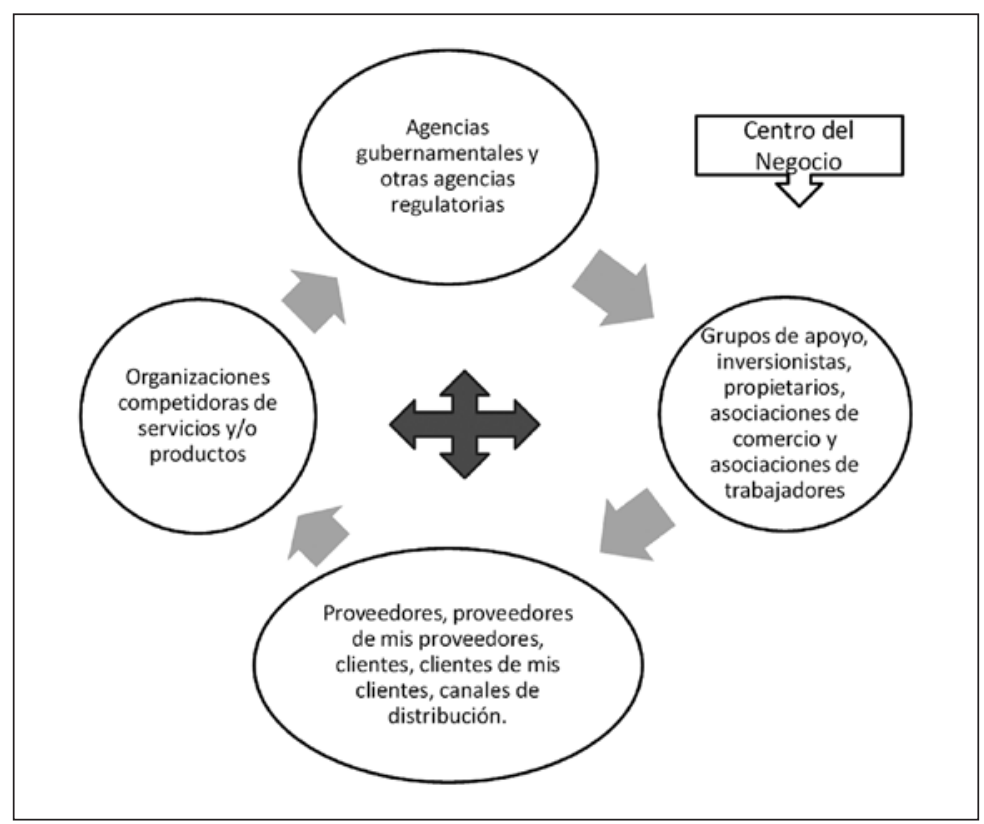

Ilustración 2. Ecosistema de negocios típico 3

\footnotetext{
$3 \quad$ Fuente: Moore (1996).
} 


\section{Ecosistema co-evolutivo}

Los elementos analizados, modelos biológicos que permiten entender los procesos de trabajo en equipo y las diferentes formas organizacionales administradas de acuerdo a sus necesidades, permiten proponer un modelo de gestión de integración empresarial que se fundamenta en un esquema eco sistémico.

En donde se puede apreciar el organismo individual como una empresa, la población como el grupo de empresas características de un sector que pueden o no estar localizadas geográficamente en un clúster o clúster virtual (Navarrete et al., 2009), la comunidad como el conjunto de organizaciones que prestan a poyo a la población y finalmente el ecosistema como toda la organización económica y social en la cual interactúa la empresa y el grupo de estas y que se convierte en su medio ambiente.

Para la gestión de la organización, la población y la comunidad se plantea el modelo de gestión sistémica, el cual permite entender el sistema como un todo y analizar sus diferentes características, es así como el proceso administrativo se hace en cada una de las organizaciones (individuos) pero a su vez se realiza para el conglomerado establecido como un nuevo individuo complejo (cooperón). Su proceso de creación sigue el ciclo de vida, siguiendo el esquema previsto (adaptado de Moore, 1996) en la ilustración 3.

En el modelo planteado, las generadoras del proceso administrativo son las intenciones de las empresas participantes. De acuerdo con ello se realiza la planeación de la gestión administrativa dejando muy claro cuáles serán los proyectos puntuales en los cuales trabajará el nuevo grupo organizacional. En el proceso de planeación de los esquemas de integración es muy importante establecer el tipo de interacción que tendrán las organizaciones, los objetivos organizacionales particulares y conjuntos, los insumos y las variables de información externas e internas. De acuerdo con ello se realiza la ejecución (encargada de la producción, distribución de bienes y servicios) de la investigación tecnológica o de las actividades previstas en la fase anterior y

Ilustración 3. Proceso de gestión del sistema de integración empresarial ${ }^{4}$

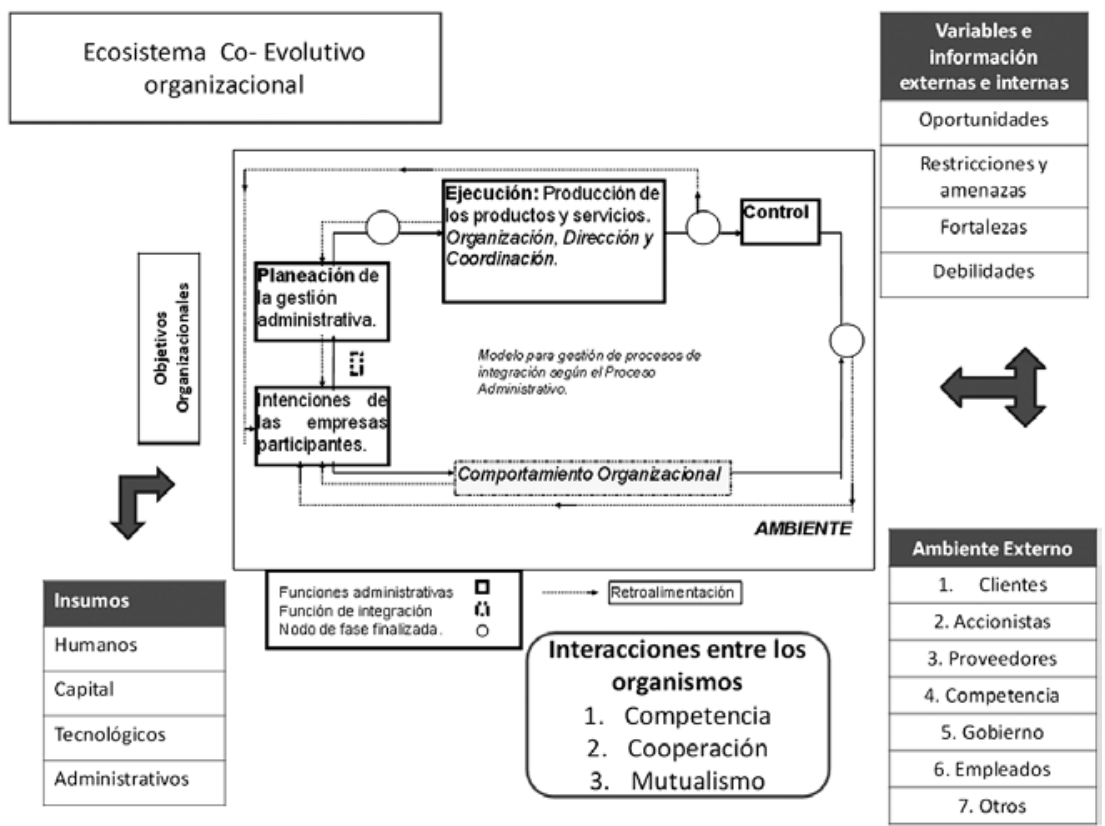

4 Fuente: Montoya (2010). 
siguiendo con el proceso se llega a los indicadores de control.

En dicho modelo, las líneas seguidas representan el flujo de información y entrada mientras que las punteadas representan el flujo de retroalimentación. Su disposición es la siguiente: i) entre las intenciones de las organizaciones participantes y la planeación estratégica y por objetivos, de doble vía; ii) entre las organizaciones participantes y la función de integración organizacional, de doble vía; iii) desde planeación a ejecución7, de doble vía; iv) de ejecución a control, sin retroalimentación; v) desde control al nodo de finalización de control, sin retroalimentación; y vi) desde la función de integración al nodo de finalización de control, sin retroalimentación.

También se tienen dos retroalimentaciones especiales:

- Retroalimentación de gestión: Entre el nodo de finalización de control y la información de las empresas participantes. Esta retroalimentación busca que los informes producidos por los tres tipos de controles sean conocidos por todos los participantes a fin de que estos últimos puedan establecer nuevas directrices en la evolución de la organización.

- Auto retroalimentación: Dado que los modelos de integración, poseen una estructura de autogestión, es claro que los directos beneficiados con un cumplimiento exitoso de la etapa de ejecución serán los mismos miembros, quienes sentirán de inmediato sus efectos y de esta forma se estarán retroalimentando.

Finalmente, el modelo debe observar el entorno en el cual la nueva forma organizativa opera, estos son los marcos jurídicos, sociales, culturales, tributarios y ambientales como contexto en el cual funciona la gestión, algunos de ellos se pueden encontrar en el recuadro de ambiente externo.
El modelo requiere unos agentes ("organismos"), empresas se relacionan por competencia, cooperación y mutualismos, los cuales se reúnen con un fin especifico, un proyecto determinado y por la posibilidad de tener un objetivo común, el cual puede ser un nuevo mercado, solucionar un problema de competitividad, el desarrollo de una nueva tecnología, para ello requiere variables e información externas e internas, que proporcionen información sobre el entorno, tales como oportunidades, restricciones y amenazas y les señale sus fortalezas y debilidades internas, también se requiere de insumos, en un modelo ecosistémico son energía, materias e información, para un ecosistema, organizacional estos son materia primas, información y recursos (financieros, tecnológicos, administrativos entre otros), finalmente debe valorar el ambiente externo en donde interactúan clientes, accionistas, proveedores, competencia, gobierno y los empleados.

Los gerentes encargados del compromiso de alcanzar modelos de integración empresarial, requieren habilidades especiales para alcanzar sus objetivos, los cuales les permitirán adaptarse a grandes retos tales como globalización, mejor conocimiento de los clientes, clientes virtuales en cualquier lugar del mundo y ello demanda "lograr cambios sostenibles en el largo plazo, como resultados del cambio a nivel individual; a su turno estos cambios individuales dependen de las estrategias de desarrollo humano que se adopten.[...] [se necesitan] cuadros directivos con apertura mental hacia nuevos mercados, nuevos procesos, nuevas tecnologías y nuevas formas de organización, típicamente, esto ha implicado la búsqueda y promoción de individuos que han trabajado en diversas áreas de la información, que cuentan con una formación generalista" (Cárdenas, 1998).

Estos gerentes deben demostrar la capacidad de aprender más que la de saber (Serna, 1998) y para ello los gerentes deberán tener nuevas cualidades

5 El mercado ha presionado la competitividad de las empresas, que ahora pueden disponer de sus propios empleados o de los de otras compañías especializadas de mayor competitividad a través de los contratos, las maquiladoras, etc. Es por esto que en últimas sólo se considera como fase a la Ejecución para que el administrador pueda libremente adoptar el propósito que aumente su efectividad. No por esto deja de ser substancial la coordinación, la dirección y la organización, que serán de vital trascendencia para el gerente en el manejo de su personal y recursos. 
tales como un liderazgo transformador, con tres principales características: Relaciones humanas en el trabajo, carisma y calidad humana, personas motivadoras con el ejemplo. Deben saber rodearse bien, con criterios para seleccionar su personal, alta delegación y la posibilidad de permitir errores en sus colaboradores porque saben que con ello su gente aprende; tienen visión del futuro, saben establecer prioridades, se anticipan y son proactivos frente al entorno. Trabajan con sentido de equipo, complementados con tres aspectos: la integridad personal, la capacidad innovadora ante una crisis y el trabajo por objetivos. Estos gerentes toman rápidamente decisiones en los momentos difíciles, buscan nuevas oportunidades y aceptan el cambio de manera creativa, plantean objetivos altos y estimulantes.

Este nuevo gestor de organizaciones debe ser investigador y diseñador. Para Senge (1998) la nueva visión del liderazgo en las organizaciones inteligentes se centra en tareas más sutiles e importantes, los diseñadores son líderes y maestros; son responsables de construir organizaciones donde la gente expande continuamente su aptitud para comprender las complejidad, clasificar la visión y mejorar los modelos mentales compartidos, siendo responsables de aprender.

Scholtes (1999) indica que se deben revaluar las capacidades de los gerentes que estaban centradas en la motivación, la delegación y los procesos de reingeniería, y empezar con nuevas capacidades, estas son:

- La habilidad para pensar en términos de sistemas y saber cómo dirigir estos sistemas.

- La habilidad para comprender la variabilidad del trabajo en la planeación y en la solución de problemas.

- Entender cómo se aprende, se desarrolla, mejora, y dirige el verdadero aprendizaje y mejoramiento.

- Entender a las personas y la razón de que se comporten como lo hacen.

- Entender la interdependencia e interacción entre sistemas, variación, aprendizaje y comportamiento humano. Saber de qué manera cada uno de estos aspectos afecta a los demás.
- Dar visión, significado y orientación a la organización.

- La disponibilidad de la información, La información puede aparecer simultáneamente en tantos lugares como sea necesario: la información genética se encuentra presente en todas las unidades, así como debe estarlo en todas las personas de la organización, a su libre disposición. (Montoya, 1999; Jiménez et al., 2009).

Los líderes necesitan apreciar la importancia de las buenas preguntas y desarrollar un instinto para formularlas, en especial la capacidad de desarrollar proyectos puntuales para que todas las organizaciones puedan trabajar con un norte común. Estos gestores de redes deben entender y aplicar el principio Holo gramático, en el cual cada producto o servicio dentro de las la organizaciones demuestra su estructura y procesos y en el cual cada persona de la organización puede satisfacer las necesidades del entorno y conocer toda la información necesaria para tomar decisiones inmediatamente (teniendo en cuenta su función y su responsabilidad). Con el principio anterior se presenta alta delegación, sus empleados deben tener alta confianza - según Fukuyama (1995),con los miembros de su equipo y con los empleados de las empresas que conforman el grupo de empresas integradas, la capacidad de una empresa de pasar de grandes estructuras jerárquicas a redes flexibles de empresas pequeñas dependerá del grado de confianza que es finalmente, la expectativa que surge dentro de una comunidad de comportamiento normal, honesto y cooperativo, basada en valores y normas comunes y compartidas - y esta debe ejercer de forma verdadera, con objetivos claros, constantes y ennoblecedores.

Las organizaciones deben contar con grupos interdisciplinarios y por proyectos, esta variedad de conocimientos permite extender las posiciones y visiones de cada una de las áreas y genera el respeto por el conocimiento de otros.

No existen relaciones de poder, existen relaciones de colaboración y mutua ayuda, los gestores deben aplicar la co-gerencia entre todos, el trabajo es de todos y todos deben responder por su éxito y logro. Por lo general deberá haber un líder o coordinador 
y todos los demás prestan su colaboración. La organización de este tipo de organizaciones es matricial por proyectos o por clientes, donde cada trabajador y co-creador de la integración tiene en primer lugar una responsabilidad y un reto como coordinador del proyecto, lo que le permite obtener su propia satisfacción y motivación y tiene el apoyo de sus colegas, dando confianza y seguridad. Todos trabajan en una misma dirección, porque comparten los valores y el direccionamiento de la empresa.

Las personas son tenidas en cuenta individualmente y su remuneración es por una parte, por desempeño y logro individual y también por otra por su desempeño colectivo. El grupo se encarga de organizar las tareas y de determinar quienes pueden seguir en los trabajos. Se plantean relaciones de amistad, de confianza, cooperación, colaboración y sobre todo de justicia. No existen las falsas competencias, ya que todos conocen la información y todos tienen su recompensa física y emocional por los resultados obtenidos. Las decisiones son por consenso y se encuentran sustentadas tanto en la lógica y la razón como en la intuición, elemento clave de las negociaciones.

\section{Consideraciones finales}

El modelo de ecosistemas organizacionales, al aplicar la metáfora biológica permite trasladar conceptos de cooperación e integración a las organizaciones, demostrando que cuando las organizaciones son vulnerables por su tamaño, condición o mercado, un grupo trabajando integradamente puede subsanarlas y de esta manera crear un súper organismo (dentro del ecosistema) que proporcione ventajas no sólo co-evolutivas sino de apoyo y protección.

\section{Referencias}

Bejarano, J. (1995). La competitividad en el sector agropecuario. En: Cuadernos de desarrollo agropecuario Fundagro. Septiembre, Bogotá.

Becker, F. (2007). Organizational ecology and knowledge networks. En: California Management Review, 49 (2) Winter.

BID - Banco Interamericano de Desarrollo (2002). Guía operativa para programas de competitividad para la pequeña y mediana empresa. Serie de buenas prácticas del departamento de desarrollo sostenible: 1-31.
Boquera, M. (2005). Las metáforas en textos de ingeniería civil: estudio contrastivo español-inglés. Tesis doctoral. Facultad de filología. Departamento de teoría de los lenguajes. Universidad de Valencia, España.

Cárdenas, M. (1998). Desarrollo humano y competitividad. La principal ventaja de las organizaciones es su capacidad de aprender. En: Periódico Portafolio. Gerencia Siglo XXI. Lunes 20 de julio.

CEPAL - Comisión Económica para América Latina y el Caribe (2001). Elementos de competitividad sistémica de las pequeñas y medianas empresas (PYME) del istmo centroamericano. CEPAL. Disponible en: http://www.eclac.cl/publicaciones/ mexico/9/lcmex1499/1499.pdf

Cerdan, C. (2003). Lecciones aprendidas sobre cadenas productivas como estrategias de desarrollo. Medellín.

Chavarría, H. (2002). Competitividad De Cadenas Agroalimentarias. Costa Rica, IICA No. 1.

Conferencia de las Naciones Unidas sobre Comercio y Desarrollo - UNCTAD (2010). CEB inter-agency cluster on trade and productive capacity. Disponible en: http://www.unctad.org/ templates/startpage .asp?intitemid $=4793 \&$ lang $=1$

Conzanelli, G. (2004). El desarrollo local. Cámara de Comercio de Bogotá, Bogotá.

Euler, R. (2008). Historia y evolución del pensamiento científico. México.

Farah, M. (2004) Pequeña empresa y competitividad. Curitiba, Juruá.

Foro Económico Mundial - FEM (2010). Anual Report 2009-2010. Disponible en: http://www.weforum.org/reports

Fukuyama, F. (1995). Trust: the social virtues y the creation of prosperity. The free press. Nueva York.

Garzón, R. (2005) Programa sobre operaciones colectivas entre empresas. Convenio BID- Atn/Me 7856 Caracas. Disponible en: www.iberpymeonline.org/venezuela130705/ricardogarzon.pdf

Guerrieri, P. \& Pietrobelli, C. (2003) Industrial districts' evolution and technological regimes: Italy and Taiwan. Universita' Di Roma 'La Sapienza', Rome, Italy, Technovation XX.

International Institute for Management Development - IMD (2010). World competitiveness yearbook. Disponible en: http://www. imd.org/research/publications/wcy/index.cfm

Jiménez, C., Castellanos, O. \& Montoya, A. (2009). Manufactura biológica e inteligente: atributos de la vida, aplicados al desarrollo tecnológico. En: Revista Ingeniería e Investigación, 29 (2): 127-134.

Kothandaraman, P. \& Wilson, D. (2001). The future of competition value-creating networks. Industrial Marketing Management Review.

Lakoff, G. \& Johnson, M. (1980). Metaphors We Live By. Chicago: The University of Chigago Press.

Lakoff, G. \& Johnson, M. (1999). Philosophy in the flesh. The Embodied Mind and Its Challenge to Western Thought. Nueva York: Basic Books.

Maya, G. (2005). Alternativas de desarrollo empresarial para la pequeña y mediana empresa en Colombia. Universidad de California.

Mincomercio (2010). Programa de productividad y competitividad. Disponible en: http://www.mincomercio.gov.co/econtent/newsdetail.asp?id=5499\&idcompany $=23$.

Mintzberg, H. (1995). La estructuración de las organizaciones. Ariel Economía, Barcelona. 
Montoya, A. (1999) Gerencia genética. Universidad Nacional De Colombia, Bogotá.

Montoya, A. (2010). Gestión de sistemas de integración empresarial bajo una perspectiva biológica. Universidad Nacional de Colombia, Bogotá. Disponible en: http://www.bdigital.unal.edu. co/2140/1/gesti\%c3\%93n_de_sistemas_de_integraci\%c3\%93n_ empresarial.pdf

Montoya, A., Montoya, I. \& Castellanos, O. (2008). De la noción de competitividad a las ventajas de la integración empresarial. En: Revista Facultad de Ciencias Económicas: Investigación y Reflexión, 16 (1): 59-70.

Montoya, A., Castellanos, O. \& Montoya, I. (2009a). Aproximación de los modelos biológicos a la comprensión de las organizaciones. En: XIII Congreso Internacional de Investigación en Ciencias Administrativas, ACACIA. La administración frente a la globalización: gobernabilidad y desarrollo, Universidad Autónoma Metropolitana - UAM, México D.F.

Montoya, A., Montoya, I. \& Castellanos, O. (2009b) Modelos de ecosistemas como alternativa para las soluciones medio ambientales, estudio de caso. En: XIII Congreso Internacional de Investigación en Ciencias Administrativas, ACACIA. La administración frente a la globalización: gobernabilidad y desarrollo, Universidad Autónoma Metropolitana UAM, México D.F.

Montoya, A., Montoya, I. \& Castellanos, O. (2010a) La metáfora organizacional: alternativa de entendimiento procedente de otras ciencias. En: Revista Facultad de Ciencias Económicas: Investigación y Reflexión, Vol. XVIII (1): 75-86.

Montoya, A., Montoya, I. \& Castellanos, O. (2010b) Situación de la competitividad de las pyme en Colombia: elementos actuales y retos. En: Agronomía Colombiana, 28(1): 107-117.

Montoya, A., Montoya, I. \& Castellanos, O. (2010c). Características de una aproximación biológica para el estudio de mecanismos de integración empresarial. En: XIV Congreso Internacional de Investigación en Ciencias Administrativas. El rol de las ciencias administrativas en el desarrollo social tecnológico de Monterrey. Monterrey, México.

Moore, J. (1993). Predatos y prey: A new ecology of competition. En: Harward Business Review, May- June. Reprint 933309.

Moore. J. (1996). Death of competition. The age of business ecosystems. En: Fortune, 133 (7): 1. John Willey \& Sons. USA.

Moore, J. (2005). Business ecosystems and the view from the firm. The Antitrust Bulletin/Fal.
Morgan, G. (1991). Imágenes de la organización. Alfaomega, México. Navarrete, D. Montoya, A. \& Montoya, I. (2009). Clusters como un modelo en el desarrollo de los negocios electrónicos. En: Revista Innovar. Universidad Nacional de Colombia, Bogotá.

Ochoa, D. \& Montoya, A. (2010). Consorcios microbianos: una metáfora biológica aplicada a la asociatividad empresarial en cadenas productivas agropecuarias. En: Revista Facultad de Ciencias Económicas: Investigación y Reflexión, XVIII (2).

Ortiz, F. \& García, M. (2003). Metodología de la investigación: el proceso y sus técnicas. México: Limusa.

Porter, M. (1990). La ventaja competitiva de las naciones. México: Editorial Cesa.

Porter, M. (2000). Cómo influyen las fuerzas de la competencia en la formación de una estrategia. En: Colección documentos IICA, Serie de competitividad, No. 17. Bogotá, Diciembre.

Rivas, L. (2002). Nuevas formas organizacionales. En: Revista Estudios Gerenciales. Universidad ICESI. Cali, Colombia.

Salazar, A. \& Maggiorani, R. (2005). Conceptos de administración, IUPSM, Merida, España.Scholtes, Peter. 1999. Como Liderar: Hacer Que Las Cosas Sucedan; Lograr Que Las Cosas Se Hagan. Mc Graw Hill, Santafé De Bogotá.

Senge, P (1998). La quinta disciplina: cómo impulsar el aprendizaje en la organización inteligente (3a ed. en español). Buenos Aires: Granica.

Scholtes, P (1999). Cómo liderar: hacer que las cosas sucedan; lograr que las cosas se hagan. Bogotá: McGraw-Hill Interamericana.

Serna, H. (1998). Los restos de la gerencia empresarial Colombiana. En: Periódico Portafolio. Lunes 20 de julio, p.14.

Sistema Nacional de Competitividad- SNC. (2008). Política nacional de competitividad y productividad. Conpes 3527. Disponible en: http://www.snc.gov.co/es/institucionalidad/documents/ conpes/3527.pdf

Thompson, J. (2003). El proceso evolutivo. Fondo De Cultura Económica, México.

Velásquez, F. (2004). La estrategia, la estructura y las formas de asociación: fuentes de ventaja competitiva para las pymes colombianas. En: Revista Estudios Gerenciales, No. 093. Universidad ICESI. Cali, Colombia.

Williamson, O. (1975). Mercados y jerarquías, su análisis e implicaciones. F.C.E, México. 